This is an Open Access article, distributed under the terms of the Creative Commons Attribution licence (http://creativecommons.org/licenses/by/4.0/), which permits unrestricted re-use, distribution, and reproduction in any medium, provided the original work is properly cited.

doi:10.1017/jfm.2019.886

\title{
Solidification of liquid metal drops during impact
}

\author{
Marise V. Gielen ${ }^{1,2}$, Riëlle de Ruiter ${ }^{2}$, Robin B. J. Koldeweij ${ }^{1}$, \\ Detlef Lohse $^{1}$, Jacco H. Snoeijer ${ }^{1,3}$ and Hanneke Gelderblom ${ }^{1,3, \dagger}$ \\ ${ }^{1}$ Physics of Fluids Group, Max Planck Center Twente for Complex Fluid Dynamics, JM Burgers Center, \\ and MESA+ Center for Nanotechnology, Department of Science and Technology, University of Twente, \\ P.O. Box 217, 7500 AE Enschede, The Netherlands \\ ${ }^{2}$ ASML The Netherlands B.V., De Run 6501, 5504 DR Veldhoven, The Netherlands \\ ${ }^{3}$ Department of Applied Physics, Eindhoven University of Technology, Den Dolech 2, 5600 MB \\ Eindhoven, The Netherlands
}

(Received 24 April 2019; revised 15 October 2019; accepted 23 October 2019)

Hot liquid metal drops impacting onto a cold substrate solidify during their subsequent spreading. Here we experimentally study the influence of solidification on the outcome of an impact event. Liquid tin drops are impacted onto sapphire substrates of varying temperature. The impact is visualised both from the side and from below, which provides a unique view on the solidification process. During spreading, an intriguing pattern of radial ligaments rapidly solidifies from the centre of the drop. This pattern determines the late-time morphology of the splat. A quantitative analysis of the drop spreading and ligament formation is supported by scaling arguments. Finally, a phase diagram for drop bouncing, deposition and splashing as a function of substrate temperature and impact velocity is provided.

Key words: solidification/melting

\section{Introduction}

When a liquid metal drop impacts a substrate with a temperature below the liquid's melting point, it rapidly cools down and solidifies. For a liquid metal, the time scales involved in cooling and solidification are comparable to the impact time of the drop. As a consequence, the impact dynamics of the drop is significantly altered by solidification (Aziz \& Chandra 2000; Mostaghimi, Pasandideh-Fard \& Chandra 2002; Pasandideh-Fard et al. 2002; Mehdizadeh, Chandra \& Mostaghimi 2004; Chandra \& Fauchais 2009).

In many applications, drops solidify during the impact event. Solidifying liquid metal drops are used in three-dimensional (3-D) printing to fabricate precise structures (Vaezi, Seitz \& Yang 2013; Visser et al. 2015; Wang et al. 2016). In thermal spray coating, liquid metal drops are sprayed onto a surface to form a protective solid

$\dagger$ Email address for correspondence: h.gelderblom@tue.nl 
layer (Mostaghimi et al. 2002; Pasandideh-Fard et al. 2002; Fauchais et al. 2004). The solidification of impacting rain drops on, for example, road surfaces (Symons \& Perry 1997), aircraft (Cebeci \& Kafyeke 2003) or transmission lines (Szilder, Lozowski \& Reuter 2002) can have detrimental effects. In all these applications, it is important to understand how liquid solidification alters the impact and spreading dynamics of the drops.

Drop impact under isothermal conditions already shows a rich and complicated dynamics. Even basic properties such as the maximum spreading, fingering instability and splashing threshold are still subject of debate (see e.g. Yarin (2006) and Josserand $\&$ Thoroddsen (2016)). Solidification of the liquid during these processes adds further complexity. The influence of solidification on drop spreading after deposition onto a substrate (i.e. with zero impact velocity) has been investigated both experimentally and theoretically (Schiaffino \& Sonin 1997a,b; Tavakoli, Davis \& Kavehpour 2014; Ruiter et al. 2017b). However, the models developed have not yet been tested for drops with a finite impact velocity.

When a water drop at room temperature impacts a sufficiently cold substrate, it first spreads out before it solidifies, cracks or fragments (Ghabache, Josserand \& Séon 2016). Only when the water is supercooled does solidification take place on the time scale of the impact event itself (Kong \& Liu 2015; Schremb et al. 2017a; Schremb, Roisman \& Tropea 2017b, 2018). In that case, solidification starts from heterogeneous nucleation at the substrate and is followed by the rapid propagation of a thin ice layer along the surface of the drop and the formation of dendrites (Schremb et al. 2018). By contrast, hot liquid metal drops impacting a substrate with a temperature below the melting point undergo an equilibrium solidification process (Fedorchenko \& Wang 2007).

The influence of solidification on the impact of (plasma-)sprayed metal drops has been studied by Mostaghimi et al. (2002), Pasandideh-Fard et al. (2002), Dhiman, McDonald \& Chandra (2007), Fedorchenko \& Wang (2007) and Chandra \& Fauchais (2009). However, in sprays, it is difficult to observe isolated impact events of drops with a controlled size and velocity. Experiments of single-drop impacts have focused on the splat morphology by top-view photography (Pasandideh-Fard et al. 1998; Aziz \& Chandra 2000; Mehdizadeh et al. 2004; Dhiman \& Chandra 2005), or self-peeling of the splat after a low-Weber-number impact (Ruiter, Soto \& Varanasi 2017a). However, the solidification-limited impact dynamics of liquid metal drops has so far remained unexplored, and a systematic quantitative study is lacking.

Here, we experimentally study tin drop impact onto a sapphire substrate with a controlled temperature below the melting point of tin. In $\S 2$ a set-up to generate isolated liquid tin drops on demand that allows for simultaneous side- and bottomview imaging is presented. The outcome of a typical experiment is described in $\S 3$. The maximum drop spreading is determined as a function of substrate temperature and a basic model to explain our findings is presented in $\S 4$. The bottom-view experiments reveal the growth of ligaments over time starting from early-time solidified corrugations. As a consequence, the number of ligaments that evolve from the rim of the drop is strongly affected by solidification, as is shown in $\S 5$. In $\S 6$ the splashing threshold of the impacting drops as a function of substrate temperature is determined quantitatively. We compare our results to previously reported findings and discuss possible extensions of our basic solidification model in $\S 7$.

\section{Experimental method}

Isolated liquid tin drops are impacted onto a sapphire substrate. The choice of sapphire as substrate material is motivated by its transparency and high thermal 


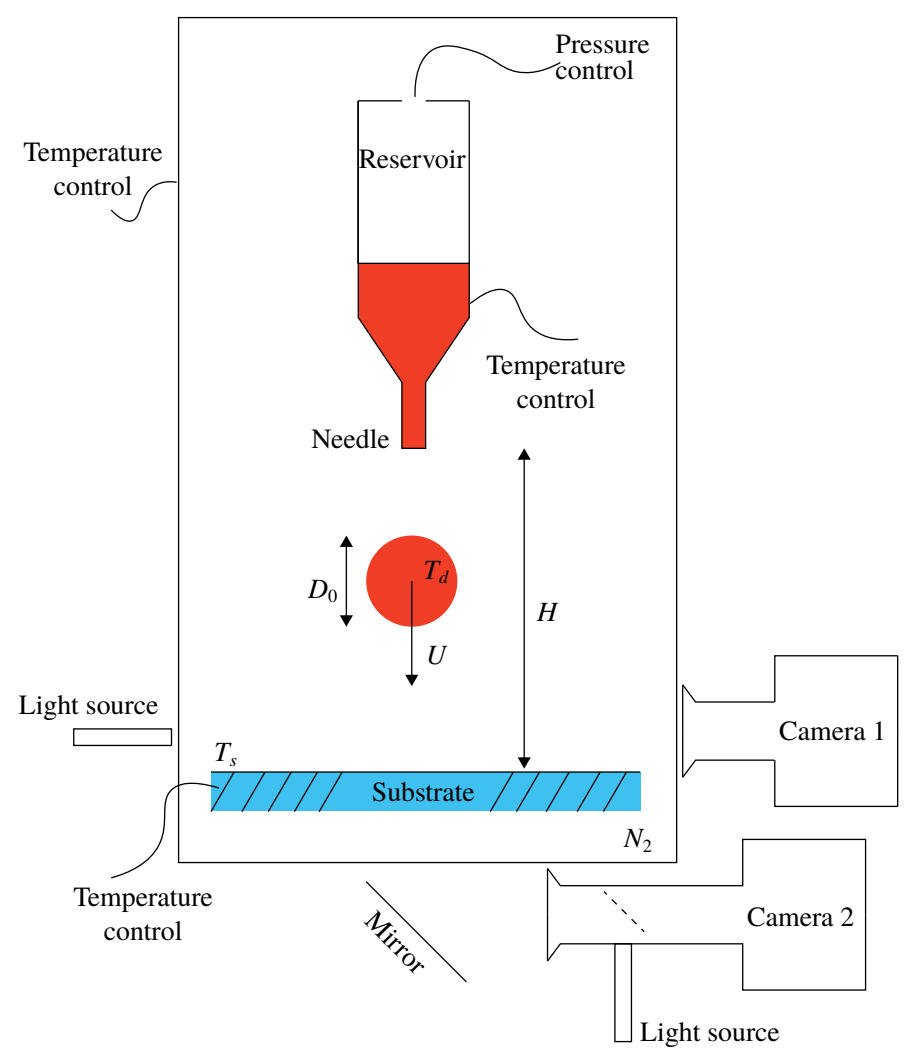

FIGURE 1. Schematic view of the drop impact set-up. Tin is contained in a reservoir that is heated to $250^{\circ} \mathrm{C}$. Upon melting, the tin fills a needle from which drops of size $D_{0}=$ $1.7 \pm 0.16 \mathrm{~mm}$ and $2.0 \pm 0.43 \mathrm{~mm}$ are pinched. The reservoir is kept at an underpressure to prevent tin leakage. To generate a drop, a short pressure pulse is given. The drop falls down under the influence of gravity. By changing the needle height $H$, the impact velocity is varied from $0.5 \mathrm{~m} \mathrm{~s}^{-1}<U<5.5 \mathrm{~m} \mathrm{~s}^{-1}$. The temperature of the sapphire substrate $T_{s}$ is controlled between $40^{\circ} \mathrm{C}$ and $250^{\circ} \mathrm{C}$. The substrate and the tin reservoir are placed inside a closed box that is kept at $250^{\circ} \mathrm{C}$ and is filled with nitrogen to prevent cooling and oxidation of the tin drops. The impact event is visualised both from the side and from below.

diffusivity (compared to other transparent materials such as glass), which allows for bottom-view visualisation of the impact event and good control of the substrate temperature. In the experiments, drop diameter $D_{0}$, impact velocity $U$ and substrate temperature $T_{s}$ are varied. An overview of the set-up is shown in figure 1.

To create single tin drops on demand, we use a method similar to the one described by Cheng, Li \& Chandra (2005) and Zhong et al. (2014). The tin is contained in a reservoir that is heated to $250{ }^{\circ} \mathrm{C}$, which is well above the melting temperature of tin $\left(T_{m}=232^{\circ} \mathrm{C}\right)$. We used $99.9 \%$ pure tin, which has (in liquid phase at $250{ }^{\circ} \mathrm{C}$ ) a density $\rho=7.0 \times 10^{3} \mathrm{~kg} \mathrm{~m}^{-3}$, surface tension $\gamma=0.54 \mathrm{~N} \mathrm{~m}^{-1}$, kinematic viscosity $v=2.6 \times 10^{-7} \mathrm{~m}^{2} \mathrm{~s}^{-1}$, thermal conductivity $k=31 \mathrm{~W} \mathrm{~m}^{-1} \mathrm{~K}^{-1}$, specific heat $c_{p}=$ $2.3 \times 10^{2} \mathrm{~J} \mathrm{~kg}^{-1} \mathrm{~K}^{-1}$ and latent heat $L=5.9 \times 10^{4} \mathrm{~J} \mathrm{~kg}^{-1}$. The bottom of the reservoir is tapered to allow for a smooth connection to the needle (Hamilton, custom-made, stainless steel, diameters 0.71 and $1.14 \mathrm{~mm}$ ). 
The pressure inside the reservoir is maintained by controllers that are situated $1 \mathrm{~m}$ higher, such that they remain at room temperature (working temperature) while the reservoir is heated. The reservoir is kept at an underpressure of $p \approx-55$ mbar to prevent leakage of liquid tin. To generate a tin drop, this underpressure is replaced by a short pressure pulse of 340 mbar (pulse duration between $0.06 \mathrm{~s}<\Delta t<0.09 \mathrm{~s}$ depending on the height of the tin column in the reservoir). The reservoir is mounted on a moveable stage of $1 \mathrm{~m}$ that allows one to change the impact height and thereby the impact velocity of the drop between $0.5 \mathrm{~m} \mathrm{~s}^{-1}<U<5.5 \mathrm{~m} \mathrm{~s}^{-1}$. The drop size is $1.7 \pm 0.16 \mathrm{~mm}$ or $2.0 \pm 0.43 \mathrm{~mm}$, depending on the needle diameter.

To ensure the liquid tin drop does not oxidise during its fall, the entire set-up is contained in a closed box filled with nitrogen gas at a slight overpressure to prevent oxygen leaks. During heating the set-up is flushed with nitrogen, while during operation the flow rate is reduced to minimise disturbances to the impacting drop. The temperature of the box is controlled at $T=250{ }^{\circ} \mathrm{C}$ to prevent cooling of the drop during its fall.

At the bottom of the closed box, a sapphire substrate (thickness $h=3 \mathrm{~mm}$, thermal conductivity $k_{\text {sub }}=27 \mathrm{~W} \mathrm{~m}^{-1} \mathrm{~K}^{-1}$, specific heat $c_{p, s u b}=763 \mathrm{~J} \mathrm{~kg}^{-1} \mathrm{~K}^{-1}$ and density $\rho_{\text {sub }}=3.98 \times 10^{3} \mathrm{~kg} \mathrm{~m}^{-3}$ ) is placed inside a temperature-controlled holder. The centre of the metal holder is open to allow for bottom-view measurements. The substrate temperature is varied between $40^{\circ} \mathrm{C}<T_{s}<250^{\circ} \mathrm{C}$. After placement, the substrate is left to equilibrate for $10 \mathrm{~min}$ to ensure that it is heated uniformly.

We measure the outcome of the impact using high-speed imaging from the side and from below. The side-view images (camera 1 in figure 1, Photron SA1.1, 30000 frames per second (f.p.s.), combined with a long-distance microscope, K2 DistaMax by Infinity Photo-Optical Company, resolution $33.8 \mu \mathrm{m} \mathrm{pixel}{ }^{-1}$ ) are taken with back-light illumination (Sumita LS-M352A); and the bottom-view images (camera 2 in figure 1, Photron SA-X2, 30000 f.p.s., combined with a Navitar $12 \times$ zoom lens, resolution $18.1 \mu \mathrm{m}$ pixel $^{-1}$ ) with coaxial illumination (Asahi MAX-303). Both cameras are synchronised to the pressure pulse that generates the drop with a time delay of $0.4 \mathrm{~s}$.

\section{Qualitative observations}

\subsection{Typical time series}

Figure 2 shows the time series of three drops impacting at similar Weber number, $W e=\rho D_{0} U^{2} / \gamma$, onto a sapphire substrate with temperatures of $(a) T_{s}=249^{\circ} \mathrm{C}$ (isothermal impact), (b) $150^{\circ} \mathrm{C}$ and $(c) 39^{\circ} \mathrm{C}$. The top row of images shows the drop impact event in side view, and the bottom row shows images in bottom view. Before impact (panel (i) in each of figure $2(a-c)$ ) the drop is not visible in bottom view since there is no contact with the substrate.

During isothermal impact (figure $2 a$, supplementary movie 1) the drop spreads smoothly over the substrate at early times (panel $a$.ii). Over time, ligaments become visible (panel a.iii), merge and grow until the drop reaches its maximum spreading (panel a.iv). As ligaments continue to appear, split and merge, their total number changes over time (Thoroddsen \& Sakakibara 1998). After reaching its maximum spreading, the drop retracts and partially bounces off the substrate while small droplets detach (panel a.v). This bounce is caused by the repelling nature of the sapphire substrate, which has a contact angle with tin of approximately $130^{\circ}$ (as measured by side-view imaging of a deposited drop).

In figure $2(b)$ and supplementary movie 2 the substrate temperature is decreased to $T_{s}=150^{\circ} \mathrm{C}$, which is below the melting temperature of tin. While the side view 
(a)
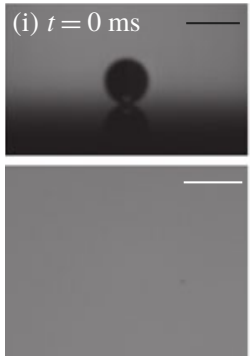

(b)
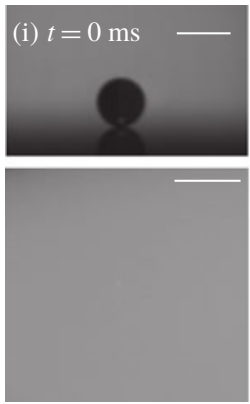

(c)
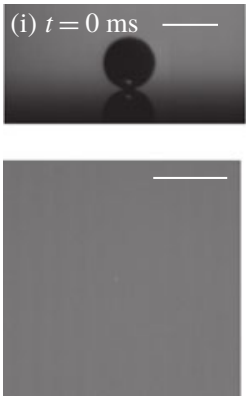
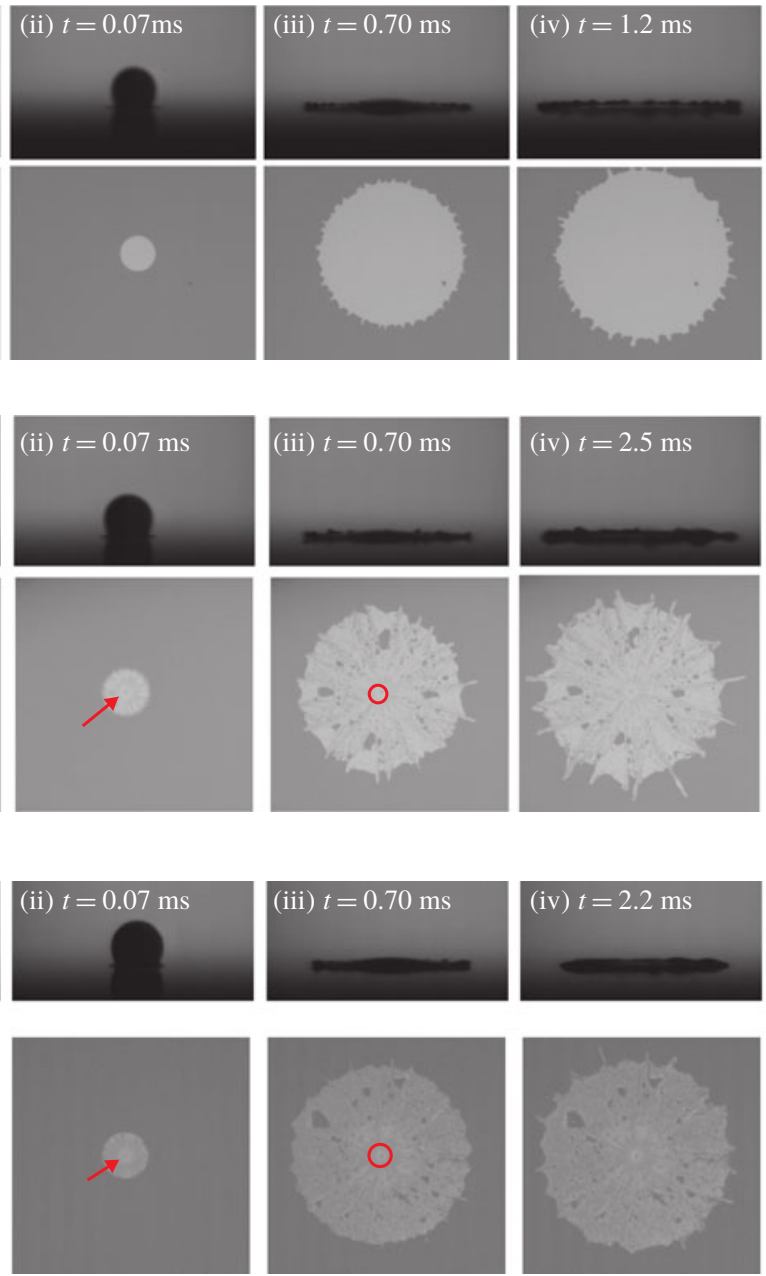
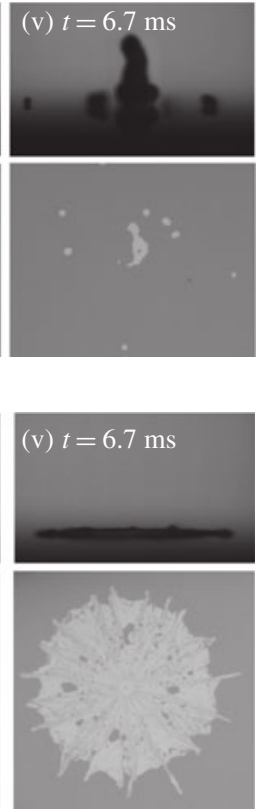

FIGURE 2. Time series of drops of similar Weber number impacting onto sapphire substrates of different temperatures: (a) $W e=281, T_{s}=249^{\circ} \mathrm{C} ;(b) W e=267, T_{s}=150{ }^{\circ} \mathrm{C}$; and $(c) W e=278, T_{s}=39^{\circ} \mathrm{C}$. The top row in each panel shows side-view images, and the bottom row shows bottom-view images. The scale bar represents $2 \mathrm{~mm}$. The drop is shown just before impact (i) and spreads over the surface in time (ii) and (iii) until it reaches its maximum spreading (iv) and retracts or remains solidified at the substrate (v). The corresponding movies can be found in the supplementary material available at https://doi.org/10.1017/jfm.2019.886.

initially looks identical to the isothermal case (cf. panels $a . i i-i v$ and $b$. ii-iv), the bottom view reveals striking differences: a grey-scale pattern appears that remains unchanged as the drop spreads and forms a clear mark of the solidification taking place at the interface with the substrate. Directly after impact a grey spot is visible at the impact centre of the drop (red arrow in panel $b . i i$; see also the close-up presented in figure $3 a$ ) surrounded by a lighter zone and grey radially outward-pointing stripes. This central grey spot is caused by the entrapment of an air bubble during first contact between the drop and the substrate (Chandra \& Avedisian 1991; van Dam \& Le Clerc 2004). In the isothermal impact experiments, this air bubble could not 
(a)

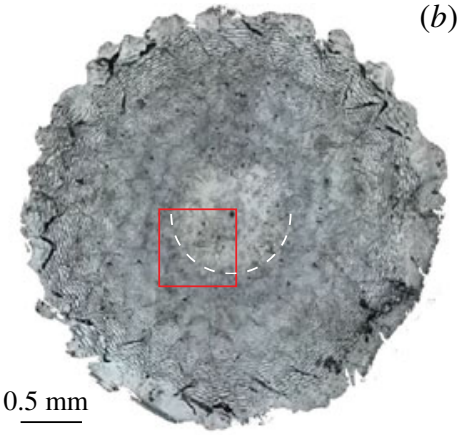

(b)

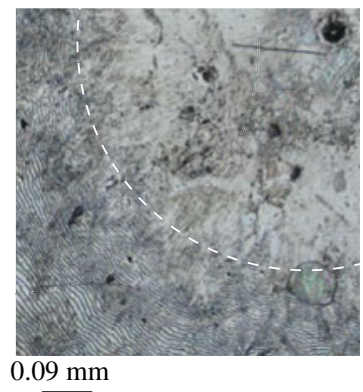

(c)

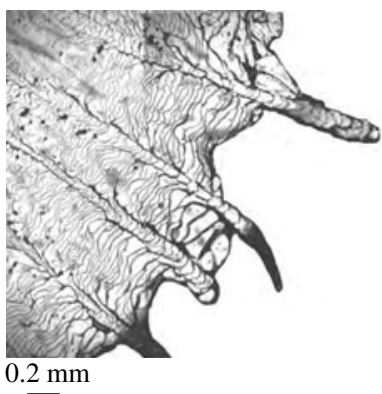

FIGURE 3. Microscope images of the solidified splat of a tin drop after impact onto a sapphire substrate. ( $a$ ) For $W e=135$ and $T_{s} \approx 50^{\circ} \mathrm{C}$. The central bubble (black spot) is surrounded by a lighter defect-free zone. The border of this zone is marked by the dashed white arc. Further outwards, a pattern of circular ridges is observed. (b) Enlargement of the area marked by the red square in panel $(a)$ to illustrate the border of the defect-free zone $D_{d} / D_{0} \approx 0.6$ (dashed white arc). (c) For $W e=448$ and $T_{s}=150{ }^{\circ} \mathrm{C}$. Close-up of the splat showing that solidified radial ligaments consist of a sequence of air ridges and can be traced back towards the centre of the splat.

be observed long enough in the bottom-view images, presumably due to pinch-off and detachment (Lee et al. 2012). The light grey zone surrounding the bubble (red circle in panel $b$.iii; see also figure $3 b$ ) has no texture. A similar defect-free zone $D_{d}$ has been observed by Ruiter et al. $(2017 a)$. These authors attributed the existence of this zone to a delay in the solidification caused by the vertical flow of hot liquid in combination with the diverging contact line velocity.

When the drop spreads further (panel $b$.iii), ligaments evolve from its edge. These ligaments immediately solidify on the substrate and elongate as time progresses while their number remains unchanged, in contrast to the isothermal case. The total number of ligaments present at maximum spread is smaller than for the isothermal impact (cf. panels $a$.iv and b.iv), as was also observed by Aziz \& Chandra (2000). In fact, a close inspection shows that the ligaments in the solidified splat can be traced all the way back to the grey stripe pattern that was already visible at an early stage (panel $b$. ii and figure $3 c$ ). The imprint of the spreading drop also shows dark grey spots. At these locations, the spreading of liquid tin is hindered by imperfections at the substrate, which results in the entrapment of air pockets. Owing to solidification of the surrounding tin, most of these air pockets remain visible at the final splat (panel $b . \mathrm{v}$ ). Solidification reduces the overall spreading velocity of the drop and the maximum spread as compared to isothermal impact. After the maximum expansion is reached, the bulk of the drop solidifies (panel $b . \mathrm{v}$ ). A retraction phase is therefore not observed and the bottom-view image no longer changes over time.

When the substrate temperature is decreased further $\left(T_{s}=39^{\circ} \mathrm{C}\right.$, figure $2 c$ and supplementary movie 3), we again observe a central air bubble (red arrow in panel $c$. ii), a defect-free zone (red circle in panel $c$.iii) and ligaments that form from a radial stripe pattern (panel $c$.iv). The number of ligaments is again smaller than for isothermal impact (figure 2a), but comparable to the experiment shown in figure $2(b)$. The maximum spreading diameter (panel c.iv) of the drop is smaller than in figure 2(b). Moreover, maximum spreading is followed by an inward-moving front (red arrow in panel $c . v$; see also supplementary movie). This self-peeling of the 
splat results from a build-up of bending stress due to shrinkage of the tin as it cools down (Ruiter et al. 2017a). Indeed, the splats of the drops that show this self-peeling are much easier to remove from the substrate after the experiment than those that do not peel off. For impacted water drops, such build-up of bending stress due to solidification has been shown to result in dramatic cracking and fragmentation events (Ghabache et al. 2016).

\subsection{Microscopy of the splat}

Splats that self-peel could be removed from the substrate for evaluation under the microscope. An example of a microscope image for an experiment with $W e=135$ is shown in figure 3(a). The black spot in the centre of the splat again corresponds to the central air bubble that is trapped in the solidified liquid. The dashed white arc marks the border of the defect-free zone. In the magnified view in figure $3(b)$, the defect-free zone and its border can be seen in more detail. Outside this zone a pattern of circular rings is observed, similar to what has been reported by Ruiter et al. (2017a). This pattern results from the entrapment of air during pinning events at the contact line: when solidification arrests the contact line, bulk liquid tin continues to spread over the substrate and forms a new contact. Each time a contact is formed, air gets entrapped, which leads to the formation of circular ridges in the splat. In this low-Weber-number experiment, radial stripes and ligaments are not observed. The drop edge, however, does show undulations with a clear wavelength from which ligaments can evolve once the Weber number is increased.

At larger $W e$, we observe both circular ridge structures, radial stripes and ligaments (see figure $3 c$ ). One clearly observes that the solidified ligaments consist of a sequence of ridges. This observation suggests that ligaments formed by bulk liquid rapidly flow outwards over previously solidified undulations of tin. Thereby, a pattern of radial stripes is formed that can be traced all the way back to the defect-free zone in the centre of the drop. Hence, the early-time solidification of undulations of the spreading drop determines the fingering pattern observed in the final solidified splat. Note that such structures were not present in the experiments by Ruiter et al. (2017a), who considered low-Weber-number impacts only.

\section{Drop spreading}

\subsection{Experiments}

We now analyse the spreading dynamics and determine the maximum spreading as a function of $U$ and $T_{s}$. At each point in time, the equivalent diameter $D$ of the area occupied by the spreading drop is determined from the bottom-view images. Figure 4(a) shows the typical dimensionless spreading diameter $\xi=D / D_{0}$ over time $t /\left(D_{0} / U\right)$ for three substrate temperatures at a similar impact velocity: $T_{s}=249^{\circ} \mathrm{C}$ (which is above $T_{m}$, red dots), $150^{\circ} \mathrm{C}$ (below $T_{m}$, yellow dots) and $39^{\circ} \mathrm{C}$ (blue dots). For each curve, the moment in time when the drop diameter reaches $\xi=0.9 \xi_{\max }$ is marked by an arrow and labelled as $t_{90}$. As shown in figure $4(b), t_{90} /\left(D_{0} / U\right)$ is of order unity, though it increases slightly with increasing substrate temperature and increasing Péclet number.

When the substrate temperature is above the melting point, no solidification takes place (red dots in figure $4 a$ ). The drop spreads to a maximum diameter $\xi_{\max }=D_{\max } / D_{0}$, retracts and bounces off the substrate. We refer to this case as 'isothermal spreading'.

At early times $\left(t \ll D_{0} / U\right)$ all drops follow the isothermal spreading curve. As time progresses, the spreading of drops on the colder $\left(150^{\circ} \mathrm{C}\right.$ and $\left.39^{\circ} \mathrm{C}\right)$ substrates slows 

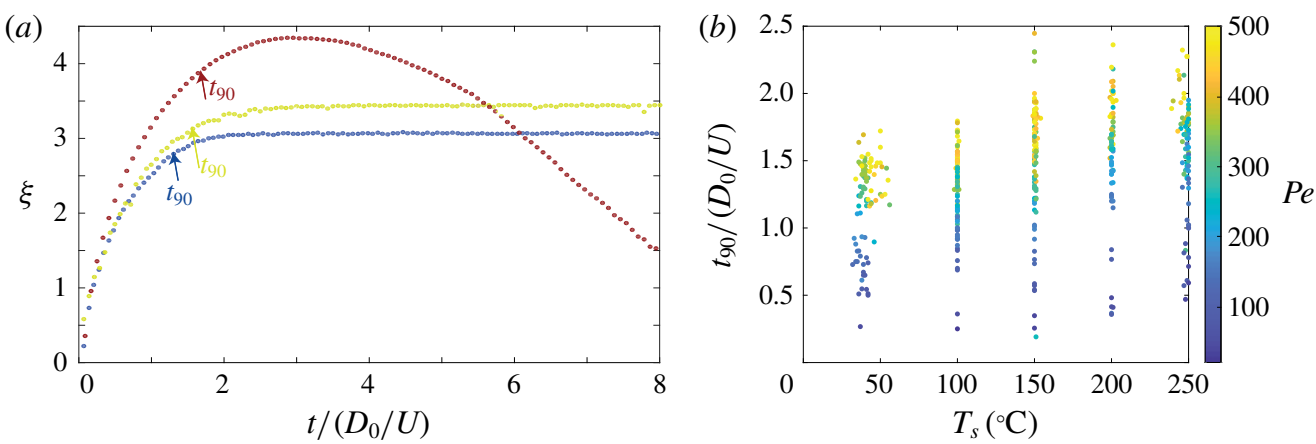

FIGURE 4. (a) Drop spreading factor $\xi=D / D_{0}$ as a function of time $t /\left(D_{0} / U\right)$ for different substrate temperatures at similar impact velocity and size: $T_{s}=249^{\circ} \mathrm{C}, U=$ $3.9 \mathrm{~m} \mathrm{~s}^{-1}, D_{0}=1.6 \mathrm{~mm}$ (red dots); $T_{s}=150^{\circ} \mathrm{C}, U=3.5 \mathrm{~m} \mathrm{~s}^{-1}, D_{0}=1.7 \mathrm{~mm}$ (yellow dots); and $T_{s}=39^{\circ} \mathrm{C}, U=3.5 \mathrm{~m} \mathrm{~s}^{-1}, D_{0}=1.7 \mathrm{~mm}$ (blue dots). The arrows mark the time $t_{90}$ at which the spreading diameter reaches $90 \%$ of its maximum value. (b) Time $t_{90} /\left(D_{0} / U\right)$ as a function of $T_{s}$ for different values of the Péclet number $P e=D_{0} U / \alpha$ (colour bar).

down and comes to rest before the retraction phase is reached. Hence, $\xi$ remains at its maximum value $\xi_{\text {max }}$. The colder the substrate, the sooner the spreading deviates from isothermal spreading, the smaller $t_{90}$ and the lower the value of $\xi_{\text {max }}$ achieved.

The maximum spreading diameter reached by the drops is extracted from the curves in figure 4(a). For isothermal spreading, Laan et al. (2014) proposed the scaling law

$$
\xi_{\text {max }} \sim \frac{P^{1 / 2}}{A+P^{1 / 2}} R e^{1 / 5},
$$

where $A=1.24$ is a fitting constant and impact parameter $P=W e R e^{-2 / 5}$, with $R e=U D_{0} / v$ the Reynolds number. In a large proportion of our experiments, $P=O(1)$, which implies that we are at the cross-over between the capillary and viscous spreading regimes and that we should use the full Padé function to describe our data (Laan et al. 2014). Clearly, given the limited range of data, one could also fit $\xi_{\max } \sim R e^{1 / 5}$, but for consistency we retain (4.1). In figure 5 we show the maximum dimensionless spreading $\xi_{\max }$ as a function of impact parameter $P$ for different substrate temperatures, where we interpret $P$ as the dimensionless impact velocity (control parameter of the experiment) and $\xi_{\max }$ as the response of the system. For isothermal impact, our data agree well with (4.1) when a prefactor of 0.9 is used. This prefactor is somewhat smaller than the prefactor of 1.0 obtained by Laan et al. (2014). We attribute this small discrepancy to the fact that we determine the spreading diameter from the equivalent area covered by the drop in the bottom-view images, thereby correcting for ligament formation, whereas Laan et al. (2014) used side-view images.

For substrate temperatures below $T_{m}$, the maximum spreading is reduced, in particular for larger $P$ and lower $T_{s}$ (see figure 5). For $T_{s} \approx 200{ }^{\circ} \mathrm{C}$, which is just below $T_{m}$, the maximum spreading is close to its isothermal value and only deviates a little for impact parameters larger than $P \approx 6$. By contrast, for a drop with $T_{s} \approx 50{ }^{\circ} \mathrm{C}$ and $P=10$, the maximum spreading decreases from $\xi_{\max }=5.2$ (isothermal) to $\xi_{\max }=3.3$, which is a reduction of $37 \%$. 


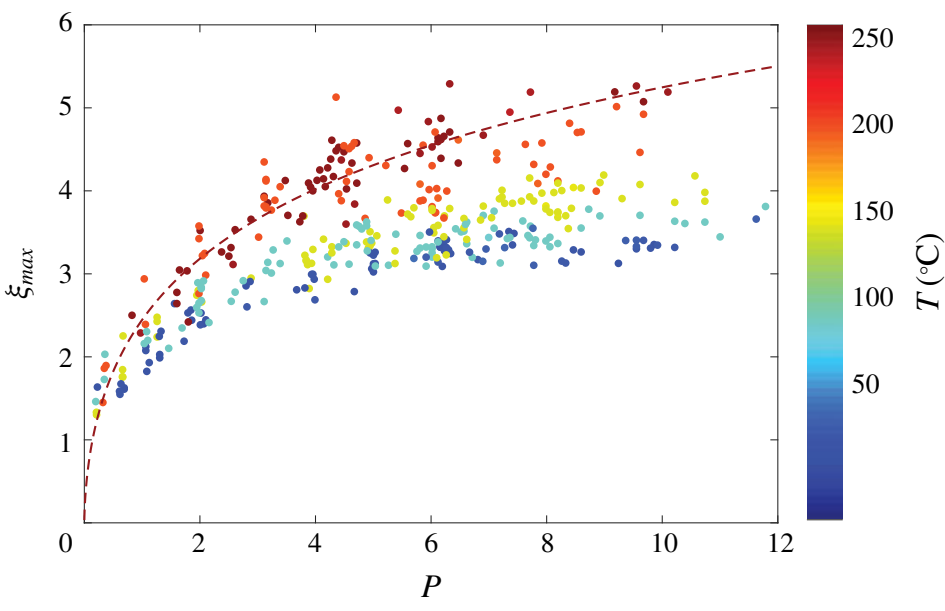

FIGURE 5. Maximum spreading $\xi_{\max }$ as a function of impact parameter $P=W e R e^{-2 / 5}$ for different substrate temperatures: $T_{s} \approx 250{ }^{\circ} \mathrm{C}$ (red dots), $T_{s} \approx 200^{\circ} \mathrm{C}$ (orange dots), $T_{s} \approx$ $150{ }^{\circ} \mathrm{C}$ (yellow dots), $T_{s} \approx 100^{\circ} \mathrm{C}$ (light blue dots) and $T_{s} \approx 50^{\circ} \mathrm{C}$ (dark blue dots). The dashed red line corresponds to the model (4.1) for isothermal spreading by Laan et al. (2014) with a prefactor of 0.9 .

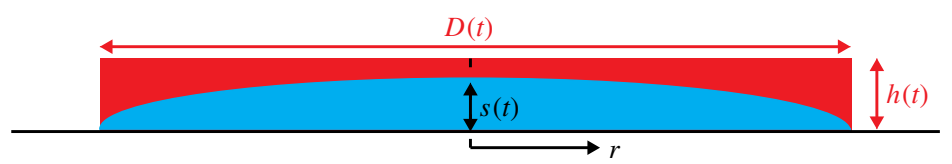

FIGURE 6. Schematic view of the growth of the solidified layer inside the spreading drop. The drop is assumed to have a pancake shape (in red) with height $h(t)$ and diameter $D(t)$. A solidified layer with thickness $s(t)$ starts growing from the moment the drop contacts the substrate. Arrest occurs when the solidified layer reaches the pancake thickness and bulk liquid can no longer overflow the contact line: $s(t)=h(t)$. Assuming heat transfer in the radial direction $r$ is negligible, one obtains a square-root dependence of the solidified layer on $r$ (see text).

\subsection{Model for solidification-limited spreading}

To explain the behaviour observed in figure 5, we derive a scaling relation for drop spreading in a regime where solidification is the limiting factor. Isothermal spreading dynamics upon impact has been modelled successfully by Eggers et al. (2010). Inspired by this model, Laan et al. (2014) derived the expression (4.1) for the maximum spreading. Eggers et al. (2010) assumed that spreading arrests when the thickness of the viscous boundary layer that grows from the substrate equals the height of the drop. Similarly, here we assume that, during solidification-limited spreading, the drop arrests when the solidified layer that grows from the cold substrate reaches the drop height, as shown schematically in figure 6. Note that until solidification takes place, the increase in kinematic viscosity of liquid tin due to its cooling is less than 5\% (Assael, Kalyva \& Antoniadis 2010) and therefore too small to account for the observed change in the drop spreading.

To find the moment of arrest, we first describe the spreading dynamics of the drop. In our experiments, arrest occurs at early times $t_{a} \sim D_{0} / U$ during the expansion phase 
(see figure $4 b$ ). In this phase, the rim still has a negligible mass and the drop shape can be approximated well by a pancake of uniform thickness $h(t)$ (Eggers et al. 2010). From mass conservation, it then follows that the pancake thickness scales as

$$
\frac{h}{D_{0}} \sim\left(\frac{D_{0}}{D}\right)^{2} .
$$

The next step is to find an expression for $D(t)$. Explicit scaling relations that cover the entire expansion phase do not exist, as the spreading diameter is the result of a complex interplay between inertial, capillary and viscous forces and eventually also wettability of the substrate (Rioboo, Marengo \& Tropea 2002; Roisman, Berberovic \& Tropea 2009; Eggers et al. 2010). We therefore look for a scaling relation that applies solely to the regime of interest $t \sim D_{0} / U$. At this time, viscous effects are confined to a tiny boundary layer of thickness $\delta / D_{0} \sim R e^{-1 / 2} \sim 10^{-2}$ and can be neglected (Riboux \& Gordillo 2014). Moreover, as $t_{a} / t_{c} \sim W e^{-1 / 2} \sim 10^{-1}$, with $t_{c}=\sqrt{\rho D_{0}^{3} / \gamma}$ the capillary time scale, we also neglect the influence of surface tension on the spreading dynamics. Since at $t_{a}$ we have $h \ll D$, we describe the pancake dynamics in the thin-film approximation. The mass and momentum conservation equations then read

$$
\begin{gathered}
r \frac{\partial h}{\partial t}+\frac{\partial}{\partial r}(r u h)=0, \\
\frac{\partial u}{\partial t}+u \frac{\partial u}{\partial r}=0,
\end{gathered}
$$

with $r$ the radial coordinate and $u$ the radial velocity. Upon integration of $(4.3 a)$ and by using (4.2), we find $u=r \dot{D} / D$. The momentum balance (4.3b) then reduces to $\ddot{D}=0$, and hence

$$
D \sim U t
$$

From (4.2) we then find the spreading dynamics of the pancake for $t \sim D_{0} / U$ to be given by

$$
\frac{h}{D_{0}} \sim\left(\frac{D_{0}}{U t}\right)^{2} .
$$

Second, we model the growth of the solidified layer from the cold substrate. Here we employ a one-dimensional heat transfer model similar to that of Pasandideh-Fard et al. (1998) and Aziz \& Chandra (2000) based on equilibrium solidification, which is justified in the regime under study (Fedorchenko \& Wang 2007). The model neglects heat transfer in the radial direction and therefore strictly applies to the dynamics of the solidification front $s(t)$ in the centre of the drop, as illustrated in figure 6 . As the drop and its surroundings are kept at approximately $T_{m}$, the heat transfer from the solidification front occurs solely through the solidified layer. In our experiments, the Stefan number of the solid

$$
\text { Ste } e_{s}=c_{p, s} \frac{T_{m}-T_{s}}{L},
$$

is typically small $\left(\right.$ Ste $\left._{s}<1\right)$ and therefore the heat transfer is assumed to be quasi-steady. The temperature dependence of the thermal properties of solidified tin is neglected and as reference the values at room temperature are used (specific heat $c_{p, s}=228 \mathrm{~J} \mathrm{~kg}^{-1} \mathrm{~K}^{-1}$, density $\rho_{s}=7310 \mathrm{~kg} \mathrm{~m}^{-3}$ and thermal conductivity 
$\left.k_{s}=67 \mathrm{~W} \mathrm{~m}^{-1} \mathrm{~K}^{-1}\right)$. Furthermore, the contact resistance between the sapphire and the tin is neglected (Aziz \& Chandra 2000), which can be justified thanks to the smooth sapphire substrates used in our experiment. The sapphire substrate will, in general, not be isothermal. The interface temperature at the contact is given by the transient convective heat transfer problem between the spreading solidifying tin drop and the conductive sapphire, and lies in between the temperature of the liquid drop and that of the substrate. To avoid this complexity, we use the substrate temperature $T_{s}$, which is a known experimental input parameter, as a reference temperature in our scaling analysis. The dynamics of the solidification front is then given by (Bejan 1993, pp. 185-188)

$$
\rho_{s} L \frac{\mathrm{d} s}{\mathrm{~d} t}=k_{s} \frac{T_{m}-T_{s}}{s},
$$

with $L$ the latent heat of solidification. From (4.7) we find the thickness of the solidified layer to scale as

$$
\frac{s}{D_{0}} \sim\left(\beta \tilde{t} \frac{S t e_{s}}{P e}\right)^{1 / 2}
$$

with $\tilde{t}=t /\left(D_{0} / U\right), \beta=\alpha_{s} / \alpha=2.1$ the ratio of solid and liquid thermal diffusivities and $P e=U D_{0} / \alpha$ the Péclet number. Solidification at each radial distance $r$ from the impact centre starts as soon as the liquid contacts the substrate. Neglecting heat transfer in the radial direction and using (4.4) and (4.8), we find the radial profile of the solidified layer to scale as $s(r, t) \sim\left[D_{0}\left(\frac{1}{2} D(t)-r\right)\right]^{1 / 2}$, as illustrated in figure 6 .

We assume that the spreading arrests as soon as there is no bulk liquid left to overflow the solidified liquid at the contact line of the drop. In terms of our scaling model, this condition translates to $h\left(t_{a}\right)=s\left(t_{a}\right)$. Combining (4.5) and (4.8) one finds for the time of arrest $t_{a} /\left(D_{0} / U\right) \sim\left(P e / \beta S t e_{s}\right)^{1 / 5}$. This scaling of $t_{a}$ motivated us to show the dependence of $t_{90}$ on the impact parameters in figure $4(b)$ using $P e$. Combining the scaling for $t_{a}$ with $D_{\max } \sim U t_{a}$ one obtains

$$
\xi_{\max } \sim\left(\frac{P e}{\beta S t e_{s}}\right)^{1 / 5} .
$$

In the regime where $P \gg 1$ and hence the isothermal data follow the scaling $\xi_{\text {max }} \sim R e^{1 / 5}$, relation (4.9) can be obtained straightforwardly: replacement of the kinematic viscosity by the diffusion coefficient of the solidification front propagation $\alpha_{s}$ Ste $e_{s}$ directly results in (4.9).

The scaling relation (4.9) is valid as long as the spreading is solidification-limited, which requires that the solidified layer grows faster than the viscous boundary layer and hence $\alpha_{s}$ Ste $_{s}>v$. In addition, surface tension forces should have a negligible influence on the maximum spreading, which requires $t_{a}<t_{c}$ or $P e / \beta S t e_{s}<W e^{5 / 2}$. When these conditions are not met and longer spreading times are considered, scaling (4.5) is no longer valid and the full spreading dynamics of the drop needs to be taken into account.

In figure 7, scaling model (4.9) is compared to the experimental data. Figure 7(a) shows the same data as in figure 5 but now plotted in terms of $P e$ for different values of Ste $_{s}$. For Ste $>0$ scaling relation (4.9) is shown with a prefactor of unity (solid lines). In addition, we also show the isothermal scaling (4.1) with prefactor 0.9 (red dashed line). Note that by rescaling (4.1) in terms of $P e$, we 


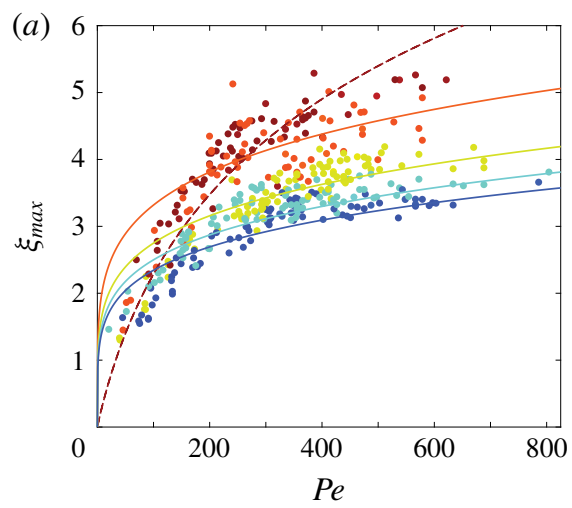

(b)

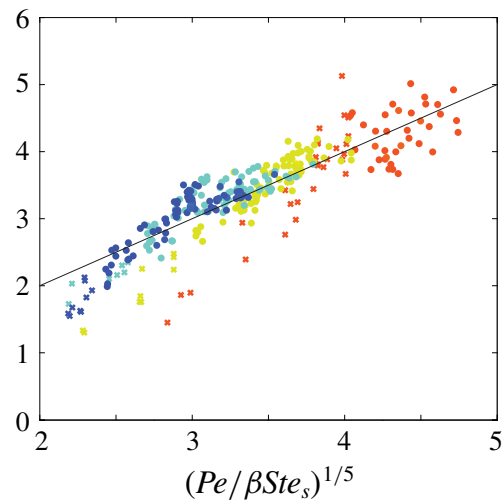

Ste $T_{s}\left({ }^{\circ} \mathrm{C}\right)$

$-0.070-250$

0.12 200

$0.32 \quad 150$

0.51

100

0.70 50

Figure 7. (a) Maximum spreading $\xi_{\max }$ as a function of $P e$ for different Ste $_{s}$ : Ste $e_{s} \approx$ -0.070 (corresponding to $T_{s} \approx 250{ }^{\circ} \mathrm{C}$, red dots), Ste $\approx 0.12\left(T_{s} \approx 200^{\circ} \mathrm{C}\right.$, orange dots), Ste $_{s} \approx 0.32\left(T_{s} \approx 150{ }^{\circ} \mathrm{C}\right.$, yellow dots $)$, Ste $e_{s} \approx 0.51\left(T_{s} \approx 100^{\circ} \mathrm{C}\right.$, light blue dots $)$ and Ste $_{s} \approx 0.70\left(T_{s} \approx 50^{\circ} \mathrm{C}\right.$, dark blue dots). The solid lines correspond to scaling (4.9) for solidification-limited spreading with a prefactor of unity. The dashed line is the isothermal spreading (4.1) rewritten in terms of $P e$ with prefactor 0.9 . (b) When rescaled in terms of $\left.(\mathrm{Pe} / \beta \mathrm{Ste})_{s}\right)^{1 / 5}$ for $\mathrm{Ste}_{s}>0$, all data collapse onto a single curve given by (4.9) with a prefactor of unity (solid line). The data points marked by crosses are not solidification-limited and therefore do not follow the master curve (see text).

introduce an explicit $D_{0}$ dependence, which results in a somewhat poorer collapse of the isothermal experimental data. Scaling (4.9) is in good agreement with the experimental data. As expected for smaller $P e$, a deviation is observed, in particular, at smaller Ste (higher substrate temperatures). For these experiments, equation (4.1) predicts a smaller spreading than (4.9) and hence solidification is not the rate-limiting factor, but viscous and/or surface tension effects dominate such that the data actually follow (4.1).

To further validate (4.9) we plot all data with $S t e_{s}>0$ rescaled in terms of $\left(\mathrm{Pe} / \beta \mathrm{Ste}_{s}\right)^{1 / 5}$ as shown in figure $7(b)$. The experiments where solidification is not rate-limiting are marked by crosses. For each $S t e_{s}$, the data corresponding to solidification-limited spreading now collapse onto a single master curve given by (4.9) with a prefactor of unity.

\section{Number of ligaments}

\subsection{Experiments}

In figure 2 we observed that solidification has a strong influence on both the development of the ligaments and their final number. To quantify these observations, we manually count the number of ligaments $N$ as a function of the Weber number for different substrate temperatures, as shown in figure 8. For isothermal impact, ligaments appear, split and merge as time progresses (see also §3). To find $N$, we therefore count the number of ligaments at the moment the drop reaches its maximum expansion, before retraction of the rim causes merger. This ambiguity in the definition of $N$ does not appear when the drop solidifies during impact and the number of ligaments is constant. 


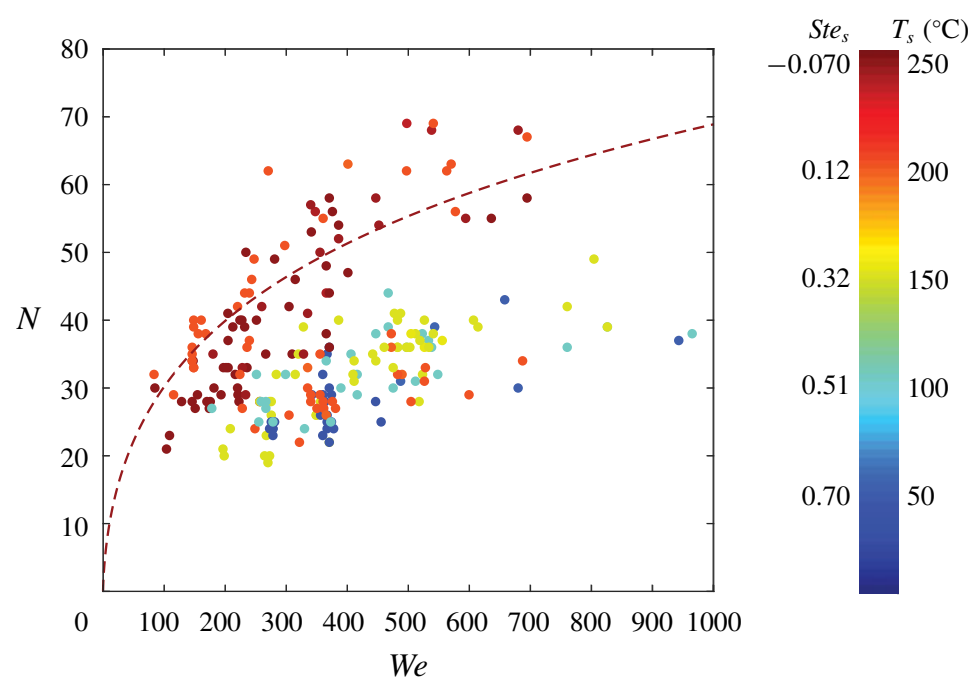

FIGURE 8. Number of ligaments $N$ as a function of We for different Ste $_{s}:$ Ste $_{s} \approx-0.070$ (corresponding to $T_{s} \approx 250{ }^{\circ} \mathrm{C}$, red dots), Ste ${ }_{s} \approx 0.12\left(T_{s} \approx 200{ }^{\circ} \mathrm{C}\right.$, orange dots), Ste ${ }_{s} \approx 0.32$ $\left(T_{s} \approx 150^{\circ} \mathrm{C}\right.$, yellow dots $)$, Ste $e_{s} \approx 0.51\left(T_{s} \approx 100^{\circ} \mathrm{C}\right.$, light blue dots $)$ and Ste $_{s} \approx 0.70$ $\left(T_{s} \approx 50^{\circ} \mathrm{C}\right.$, dark blue dots). The red dashed line shows the number of ligaments for isothermal impact as predicted by (5.1) with a prefactor of 4.3 . For $W e \lesssim 150$ no ligaments are observed.

As is clear from figure 8 , no ligaments are observed for $W e \lesssim 150$. For such small We, the impact is not violent enough and therefore the rim retracts before ligaments become apparent. For larger Weber numbers, $N$ increases with increasing We and is spread in two clouds: isothermal impacts $\left(T_{s}>T_{m}\right.$, red data points) and solidification-limited impacts $\left(T_{s}<T_{m}\right.$, yellow and blue data points). The orange data points correspond to experiments on substrates close to $T_{m}$ and appear in both clouds.

Even though each cloud has a considerable spread in $N$, we systematically observe that solidification leads to fewer ligaments. For example, $N$ (almost) halves when we compare the impact onto the coldest substrate $\left(T_{s}=50^{\circ} \mathrm{C}\right)$ to isothermal impact $\left(T_{s}=250^{\circ} \mathrm{C}\right)$.

\subsection{Qualitative explanation}

To predict the number of ligaments formed during isothermal impact, several models based on a Rayleigh-Taylor instability of the decelerating rim have been proposed (see e.g. Allen 1975; Marmanis \& Thoroddsen 1996; Aziz \& Chandra 2000; Kim, Feng \& Chun 2000; Villermaux \& Bossa 2011). In these models, complicated effects such as the splitting and merging of fingers (Thoroddsen \& Sakakibara 1998) and the influence of ambient pressure (Xu, Zhang \& Nagel 2005; Xu 2007) or surface roughness (Range $\&$ Feuillebois 1998) have been neglected. Despite these shortcomings, we now derive a modified version of the model by Aziz \& Chandra (2000) as a 'guide to the eye' to describe the isothermal data shown in figure 8 .

A Rayleigh-Taylor instability of the rim gives rise to a preferred wavenumber that scales as $k \sim \sqrt{\rho(-\ddot{D}) / \gamma}$, where $\ddot{D}$ is the acceleration of the rim. The number of ligaments is then given by $N \sim k D_{\ell}$, where $D_{\ell}$ is the spreading diameter at the moment 
of ligament expulsion. For isothermal impact, ligaments are expelled from the rim close to the maximum drop expansion, such that $D_{\ell} \sim D_{\max }$ (Aziz \& Chandra 2000). At that moment, we estimate the rim deceleration as $\ddot{D} \sim D_{\max } / t_{c}^{2}$ (Villermaux \& Bossa 2011) and hence $k \sim \sqrt{D_{\max } / D_{0}^{3}}$. Using the maximum expansion given by (4.1) we find

$$
N \sim\left(\frac{P^{1 / 2}}{A+P^{1 / 2}} R e^{1 / 5}\right)^{3 / 2} .
$$

In the limit of small $P$ (large $R e$ ) one recovers that $N \sim W e^{3 / 4}$, as was derived by Villermaux \& Bossa (2011) for drops impacting on a pillar. Scaling (5.1) differs somewhat from the one proposed by Aziz \& Chandra (2000), who used $U^{2} / D_{0}$ as an estimate for the rim deceleration as well as a different expression for $D_{\max }$. In figure 8 we show that (5.1) with a prefactor of 4.3 describes the isothermal experimental data.

For impacts on cold substrates, $N$ falls below the isothermal curve (5.1). This decrease in $N$ was already observed from top-view photography of tin splats by Aziz \& Chandra (2000), who attributed it to the smaller maximum spreading $D_{\max }$ for solidification-limited impacts. Our bottom-view images, however, allow us to follow the growth of the ligaments over time and reveal a remarkable feature: for the solidified drops, the number of ligaments is set by the early-time freezing of undulations and remains unchanged during the subsequent drop spreading, thereby forming a pattern of radially outward-pointing stripes (recall figures 2 and $3 c$ ). By contrast, for isothermal impact, these early-time undulations remain invisible and ligaments evolving from the rim split at later times (Thoroddsen \& Sakakibara 1998), thereby increasing $N$ (compare figure $2 a$.iv with figure $2 c$.ii). These two mechanisms qualitatively explain the two clouds of data for isothermal and solidification-limited spreading in figure 8 . For temperatures close to the melting temperature of tin (orange data in figure 8), both mechanisms are at play: in some drops solidification is fast enough to prevent splitting of the fingers, while in other drops new ligaments emerge at later times.

\section{Splashing threshold}

\subsection{Experiments}

An impact event may have several outcomes, depending on the impact conditions. We observed deposition of the drop, which means that the drop remains intact and adheres to the substrate after impact. Splashing occurs when secondary droplets are generated. Bouncing takes place when (part of) the drop lifts off from the substrate. In figure $9(a)$ we quantify these impact phenomena as a function of the Stefan and Weber numbers, i.e. the dimensionless substrate temperature and impact velocity.

For the isothermal impact events $\left(\right.$ Ste $\left._{s} \leqslant 0, T \geqslant T_{m}\right)$ and $W e<350$, the drop bounces. For $W e>375$, drops always splash during isothermal impact. For intermediate Weber numbers, a transition regime exists where both bouncing and splashing can occur. Drop deposition is not observed in the isothermal regime. When the substrate temperature is decreased below the melting temperature $\left(\right.$ Ste $\left._{s}=0.12, T_{s}=200^{\circ} \mathrm{C}\right)$, only a few bounces occur at low We, while most of the drops deposit and stick to the substrate. For higher Weber numbers, we again observe a transition to splashing. On even colder substrates $\left(\right.$ Ste $_{s}>0.12, T_{s}<200^{\circ} \mathrm{C}$ ), the bouncing regime completely disappears and we only observe freezing-induced sticking of the drop. The Weber number at which the transition from bouncing or deposition to splashing takes place varies with the Stefan number. Importantly, for solidification-limited impacts, there is 

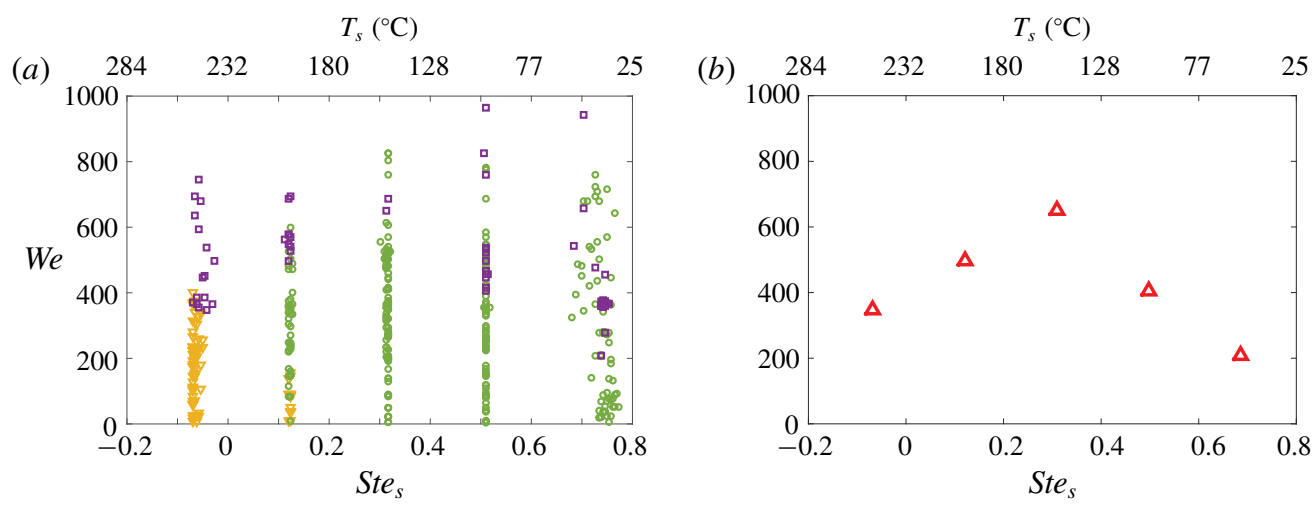

FIgURE 9. (a) Phase diagram showing the outcome of drop impact as a function of We and Ste $_{s}$. The three impact behaviours observed are bouncing (orange triangles), deposition (green circles) and splashing (purple squares). (b) Plot of the first splashing event observed as a function of We and Ste as extracted from the data in $(a)$.

no longer a sharp boundary between bouncing/deposition and splashing, but both can be observed even at the highest Weber numbers accessible by our set-up.

The splashing threshold is analysed in more detail in figure $9(b)$, where the critical Weber numbers corresponding to the first splashing event (i.e. the lowest We where splashing is observed) are plotted as a function of Ste $_{s}$. The splashing threshold shows a non-monotonic trend: it first increases up to $S t e_{s} \approx 0.3$ and then decreases again, to a value that lies below the isothermal splashing threshold.

\subsection{Qualitative explanation}

To explain the observations in figure $9(b)$, we hypothesise that the influence of solidification on the splashing threshold is twofold. First, solidification limits the spreading of the drop and the growth of ligaments as was observed in $\S \S 4$ and 5 . Consequently, one expects splashing due to ligament breakup to be suppressed, as was already suggested by Aziz \& Chandra (2000). This effect becomes stronger with increasing Ste (lower substrate temperatures), which leads to the observed increase in the critical Weber number for the first splash.

Second, rapid solidification of the ejecta sheet forms local irregularities on the substrate, thereby increasing the roughness as discussed in $\S 5$. These irregularities interact with the moving contact line and may thereby provoke a freezing-induced splash (Dhiman \& Chandra 2005; Dhiman et al. 2007; Chandra \& Fauchais 2009). This effect becomes more severe with increasing Ste $e_{s}$ such that at some point the splashing threshold decreases below that for isothermal impact. As the freezing-induced splash depends on nucleation events at the substrate, it has a stochastic nature and is not observed in each experiment.

These two competing effects, the splash suppression by solidification and the freezing-induced splash, can qualitatively explain the non-monotonic trend in the splashing threshold observed in figure $9(b)$.

\section{Discussion and conclusion}

The influence of solidification on the impact dynamics of liquid tin drops was investigated. Solidification was shown to strongly alter drop spreading, destabilisation 
through the formation of ligaments and splashing. The use of transparent sapphire substrates allowed for bottom-view imaging and provided a unique view on the solidification and impact dynamics. We now discuss our findings in light of previously reported results and reflect on possible extensions for our work.

Spreading of the liquid tin drops on cold substrates is limited by solidification rather than by surface tension or viscous effects. The spreading arrests early in the expansion phase, which results in a smaller maximum spreading. In previous studies the maximum drop spreading was modelled based on energy arguments that account for a loss in kinetic energy due to solidification (Pasandideh-Fard et al. 1998; Dhiman \& Chandra 2005; Dhiman et al. 2007). In these models, arrest was assumed to occur at a fixed time, independent of the substrate temperature. Our measurements of drop spreading over time are not consistent with this view, as we found the arrest time to decrease with decreasing substrate temperature. Instead, we derived a model for the solidification-limited spreading dynamics assuming that arrest occurs once the growing solidified layer reaches the height of the spreading drop. From the predicted time of arrest, we then found the maximum spreading diameter. In this model we used a basic description of the drop dynamics, neglecting the growth of a viscous boundary layer and surface tension forces acting on the rim of the drop. This simple model already gave a good prediction for the maximum drop spreading. To accurately describe the solidification-limited spreading dynamics, a more sophisticated model is needed; for example, an extension of the spreading model derived by Eggers et al. (2010) to account for the growth of a solidified layer. Such a model would provide a complete description of drop spreading in the presence of solidification and could account for the gradual deviation from isothermal spreading we observed in our data.

In a previous study with hexadecane drop spreading during deposition (i.e. with zero impact velocity) by Ruiter et al. (2017b), kinetic undercooling effects were used to obtain a criterion for contact line arrest. In that work, the entire drop was assumed to arrest abruptly once the liquid at the contact line reaches a critical temperature that is determined by kinetic undercooling. Our microscope images of the solidified splat revealed a sequence of circular ridges formed at the interface with the substrate. The moment the first ridge is observed, and hence the diameter of the defect-free zone, are consistent with the kinetic undercooling scenario, as explained in appendix A. This observation, together with the gradual arrest of the drop spreading, suggests that a sequence of contact line arrests occurs, each time overflown by fresh liquid from the bulk, until the bulk of the drop is solidified and the drop reaches its maximum spreading diameter.

The early-time solidification of the contact line was shown to control the late-time fingering instability: our bottom-view images revealed a pattern of radial stripes that could be traced back all the way to the defect-free zone. These stripes in turn consisted of a sequence of solidified ridges starting from the very early stages of impact. This finding is consistent with the observation by Thoroddsen \& Sakakibara (1998) that the fundamental number of disturbances forms right after the first contact of the drop with the substrate and remains unchanged during the expansion phase. For isothermal drops, these fundamental ligaments undergo splitting and merging at later times. By contrast, we have shown that, for drops impacting cold substrates, the initial undulations solidify and perturb the flow of the remaining liquid, thereby controlling the number of ligaments during the entire expansion phase. The pattern of solidified undulations will depend on the interaction between the substrate, the moving and destabilising contact line and local nucleation events. Therefore, a quantitative prediction of the fingering instability in the presence of solidification remains an open issue. 
The solidification of early-time undulations of the contact line is also a potential cause of the freezing-induced splashing that we observed. The solidified pattern locally increases the surface roughness and can thereby trigger instabilities in the spreading drop. As a result, splashing is observed at Weber numbers below the isothermal splashing threshold. By contrast, solidification can also cause deposition to occur at Weber numbers above the isothermal splashing threshold by suppressing the ligament breakup. To further quantify these effects, an experimental set-up that can achieve higher Weber numbers is required, possibly through a different drop generation method. Moreover, a clarification of the splash-suppressing and -enhancing effects of solidification requires detailed experimental data to distinguish between splashing caused by contact line destabilisation and by ligament breakup.

The quantitative prediction of the occurrence of freezing-induced splashing for solidifying drops remains challenging. On the theoretical side, one would need to account for the interaction of the ejecta sheet dynamics, the moving contact line, the growing solidification front and the underlying substrate; for example, by extending the model by Riboux \& Gordillo (2014). On the experimental side, one would need detailed data of the early stages of impact to disentangle the order in which nucleation, contact line destabilisation, arrest and sheet ejection take place.

Despite these challenges, the solidification of a liquid drop during an impact event offers a unique perspective on the impact dynamics. For example, the detailed inspection of the splat formed after impact can be used as a diagnostic tool to identify the impact conditions, such as impact speed and angle (Laan et al. 2014; Gielen 2018). Moreover, the visualisation of the impact of solidifying drops forms a powerful method to learn about isothermal impact as one literally freezes the dynamics. The freezing pattern that develops at the solid-liquid interface reveals the different stages of impact, from bubble entrapment to sheet ejection, the growth of corrugations and the development of ligaments.

\section{Acknowledgements}

We thank M. Riepen for fruitful discussions. This work is part of an Industrial Partnership Programme of the Netherlands Organization for Scientific Research (NWO). This research programme is co-financed by ASML.

\section{Supplementary movies}

Supplementary movies are available at https://doi.org/10.1017/jfm.2019.886.

\section{Appendix A}

Here, we derive a criterion for the first contact line arrest that occurs in solidifying spreading drops after impact. This first arrest determines the size of the defect-free zone, as observed in, for example, figure 3. To this end, we modify the arrest criterion for drops spreading after deposition described by Ruiter et al. (2017b) to account for the impact dynamics.

According to Ruiter et al. (2017b), spreading arrests abruptly when the solidification front catches up with the moving contact line. For rapidly moving contact lines, the velocity of the solidification front is limited by the crystallisation rate, which in turn depends on the amount of kinetic undercooling of the liquid (Amini \& Laird 2006). For a small amount of kinetic undercooling, the solidification front velocity $U_{f}$ is given by Fedorchenko \& Wang (2007)

$$
U_{f}=\kappa\left(T_{m}-T_{f}\right),
$$


with $T_{f}$ the temperature of the solidification front and $\kappa$ the kinetic coefficient. This coefficient is a property of the liquid, and is available in the literature for some metals (see e.g. Rodway \& Hunt (1991) and Fedorchenko \& Wang (2007)) but unfortunately has so far not been determined for tin.

The velocity of the contact line follows from the isothermal spreading dynamics of the drop. In our experiments the drop diameter at the first arrest $D_{d}$ is small $\left(D_{d} \ll\right.$ $D_{\max }$, see figure $\left.3 a\right)$. In this early-time kinematic regime $(t<0.1)$, surface tension and viscous effects do not yet affect the spreading dynamics and the drop diameter is given by Rioboo et al. (2002)

$$
\xi=c \tilde{t}^{1 / 2}
$$

where $\tilde{t}=t /\left(D_{0} / U\right)$ and the proportionality constant $c=\sqrt{6}$ (Riboux \& Gordillo 2014). The contact line velocity then reads

$$
U_{c l}=\frac{1}{2} U \frac{\mathrm{d} \xi}{\mathrm{d} \tilde{t}}=\frac{1}{4} c U \tilde{t}^{-1 / 2}=\frac{1}{4} c^{2} U \xi^{-1} .
$$

In line with Ruiter et al. (2017b), we assume that the contact line arrests once the velocity of the contact line equals the velocity of the solidification front, $U_{c l}=U_{f}$. Hence, arrest occurs once the temperature of the contact line is equal to the temperature of the solidification front, i.e. $T_{c l}=T_{f}$. For small contact angles, temperature gradients inside the drop are negligible such that the temperature at the contact line equals the substrate temperature $T_{c l}=T_{s}$ (Ruiter et al. 2017b). The arrest criterion is then given by

$$
U_{c l}=\kappa\left(T_{m}-T_{s}\right) .
$$

Combining (A 3) and (A 4) we obtain an expression for the defect distance as

$$
\xi_{d}=\frac{D_{d}}{D_{0}}=\frac{1}{4} c^{2} \frac{U}{\kappa\left(T_{m}-T_{s}\right)} .
$$

For the experiment shown in figure $3(a, b)$ we find $\xi_{d} \approx 0.6$ (as determined from high-resolution images). Relation (A 5) then gives $\kappa=0.034 \mathrm{~m} \mathrm{~s}^{-1} \mathrm{~K}^{-1}$, which is of the right order of magnitude for metals (Rodway \& Hunt 1991; Fedorchenko \& Wang 2007; Monk et al. 2010). Our analysis therefore provides evidence that the first contact line arrest, and hence the size of the defect-free zone, is determined by non-equilibrium crystallisation.

\section{REFERENCES}

Allen, R. F. 1975 The role of surface tension in splashing. J. Colloid Interface Sci. 51, 350-351. Amini, M. \& LAIRD, B. B. 2006 Kinetic coefficient for hard-sphere crystal growth from the melt. Phys. Rev. Lett. 97, 216102.

Assael, M. J., Kalyva, A. E. \& Antoniadis, K. D. 2010 Reference data for the density and viscosity of liquid copper and liquid tin. J. Phys. Chem. Ref. Data 39, 033105.

AzIZ, S. D. \& ChANDRA, S. 2000 Impact, recoil and splashing of molten metal droplets. Intl J. Heat Mass Transfer 43 (16), 2841-2857.

BeJan, A. 1993 Heat Transfer. John Wiley \& Sons, Inc.

Cebeci, T. \& Kafyeke, F. 2003 Aircraft icing. Annu. Rev. Fluid Mech. 35 (1), 11-21.

Chandra, S. \& Avedisian, C. T. 1991 On the collision of a droplet with a solid surface. Proc. R. Soc. A 432, 13-41. 
Chandra, S. \& Fauchais, P. 2009 Formation of solid splats during thermal spray deposition. J. Therm. Spray Technol. 18 (2), 148-180.

Cheng, S. X., Li, T. \& Chandra, S. 2005 Producing molten metal droplets with a pneumatic droplet-on-demand generator. J. Mater. Process. Technol. 159 (3), 295-302.

van DaM, D. B. \& LE ClerC, C. 2004 Experimental study of an inkjet printed droplet on a solid substrate. Phys. Fluids 16, 3404-3414.

Dhiman, R. \& Chandra, S. 2005 Freezing-induced splashing during impact of molten metal droplets with high Weber numbers. Intl J. Heat Mass Tranfer. 48 (25), 5625-5638.

Dhiman, R., McDonald, A. G. \& Chandra, S. 2007 Predicting splat morphology in a thermal spray process. Surf. Coat. Technol. 201 (18), 7789-7801.

Eggers, J., Fontelos, M. A., Josserand, C. \& Zaleski, S. 2010 Drop dynamics after impact on a solid wall: theory and simulations. Phys. Fluids 22, 062101.

Fauchais, P., Vardelle, A., Vardelle, M. \& Fukumoto, M. 2004 Knowledge concerning splat formation: an invited review. J. Therm. Spray Technol. 13, 337-360.

Fedorchenko, A. I. \& WANG, A.-B. 2007 Non-equilibrium solidification of the molten metal droplets impacting on a solid surface. Intl J. Heat Mass Transfer 50, 463-2468.

Ghabache, E., Josserand, C. \& SÉon, T. 2016 Frozen impacted drop: from fragmentation to hierarchical crack patterns. Phys. Rev. Lett. 117, 074501.

Gielen, M. V. 2018 Splashing drops. PhD thesis, University of Twente.

Josserand, C. \& Thoroddsen, S. T. 2016 Drop impact on a solid surface. Annu. Rev. Fluid Mech. 48, 365-391.

KIm, H. Y., Feng, Z. C. \& ChUn, J. H. 2000 Instability of a liquid jet emerging from a droplet upon collision with a solid surface. Phys. Fluids 12, 531-541.

KonG, W. \& LiU, H. 2015 A theory on the icing evolution of supercooled water near solid substrate. Intl J. Heat Mass Transfer 91, 1217-1236.

LaAn, N., De Bruin, K. G., Bartolo, D., Josserand, C. \& Bonn, D. 2014 Maximum diameter of impacting liquid droplets. Phys. Rev. A 2, 044018.

Lee, J. S., Weon, B. M., Je, J. H. \& FezzaA, K. 2012 How does an air film evolve into a bubble during drop impact? Phys. Rev. Lett. 109, 204501.

Marmanis, H. \& Thoroddsen, S. T. 1996 Scaling of the fingering pattern of an impacting drop. Phys. Fluids 8, 1344-1346.

Mehdizadeh, N. Z., Chandra, S. \& Mostaghimi, J. 2004 Formation of fingers around the edges of a drop hitting a metal plate with high velocity. J. Fluid Mech. 510, 353-373.

Monk, J., Yang, Y., Mendelev, M. I., Asta, M., Hoyt, J. J. \& Sun, D. Y. 2010 Determination of the crystal-melt interface kinetic coefficient from molecular dynamics simulations. Model. Simul. Mater. Sci. Engng 18 (1), 015004.

Mostaghimi, J., Pasandideh-Fard, M. \& Chandra, S. 2002 Dynamics of splat formation in plasma spray coating process. Plasma Chem. Plasma Process. 22 (1), 59-84.

Pasandideh-Fard, M., Bhola, R., Chandra, S. \& Mostaghimi, J. 1998 Deposition of tin droplets on a steel plate: simulations and experiments. Intl J. Heat Mass Transfer. 41 (19), 2929-2945.

Pasandideh-Fard, M., Pershin, V., Chandra, S. \& Mostaghimi, J. 2002 Splat shapes in a thermal spray coating process: simulations and experiments. J. Therm. Spray Technol. 11 (2), 206-217.

RAnGe, K. \& Feuillebois, F. 1998 Influence of surface roughness on liquid drop impact. J. Colloid Interface Sci. 203, 16-30.

Riboux, G. \& Gordillo, J. M. 2014 Experiments of drops impacting a smooth solid surface: a model of the critical impact speed for drop splashing. Phys. Rev. Lett. 113, 024507.

Rioboo, R., Marengo, M. \& Tropea, C. 2002 Time evolution of liquid drop impact onto solid, dry surfaces. Exp. Fluids 33 (1), 112-124.

Rodway, G. H. \& Hunt, J. D. 1991 Thermoelectric investigation of solidification of lead I. Pure lead. J. Cryst. Growth 112 (2), 554-562.

Roisman, I. V., Berberovic, E. \& Tropea, C. 2009 Intertia dominated drop collisions. I. On the universal flow in the lamella. Phys. Fluids 21, 052103. 
Ruiter, J. De, Soto, D. \& Varanasi, K. K. $2017 a$ Self-peeling of impacting droplets. Nat. Phys. 14 (1), 35-39.

Ruiter, R. De, Colinet, P., Brunet, P., Snoeijer, J. H. \& Gelderblom, H. 2017b Contact line arrest in solidifying spreading drops. Phys. Rev. Fluids 2, 043602.

Schiaffino, S. \& Sonin, A. A. $1997 a$ Motion and arrest of a molten contact line on a cold surface: an experimental study. Phys. Fluids 9, 2217-2226.

SCHIAfFino, S. \& Sonin, A. A. $1997 b$ On the theory for the arrest of an advancing molten contact line on a cold solid of the same material. Phys. Fluids 9, 2227-2233.

Schremb, M., Borchert, S., Berberovic, E., Jakirlic, S., Roisman, I. V. \& Tropea, C. $2017 a$ Computational modelling of flow and conjugate heat transfer of a drop impacting onto a cold wall. Intl J. Heat Mass Transfer 109, 971-980.

Schremb, M., Roisman, I. V. \& Tropea, C. $2017 b$ Transient effects in ice nucleation of a water drop impacting onto a cold substrate. Phys. Rev. E 95 (2), 022805-6.

Schremb, M., Roisman, I. V. \& Tropea, C. 2018 Normal impact of supercooled water drops onto a smooth ice surface: experiments and modelling. J. Fluid Mech. 835, 1087-1107.

SYMONS, L. \& PERRY, A. 1997 Predicting road hazards caused by rain, freezing rain and wet surfaces and the role of the weather radar. Meteorol. Appl. 4, 17-21.

Szilder, K., Lozowski, E. P. \& Reuter, G. 2002 A study of ice accretion shape on cables under freezing rain conditions. Trans. ASME J. Offshore Mech. Arctic Engng 124, 162-168.

Tavakoli, F., Davis, S. H. \& Kavehpour, H. P. 2014 Spreading and arrest of a molten liquid on cold substrates. Langmuir 30 (34), 10151-10155.

Thoroddsen, S. T. \& SAKAKibARA, J. 1998 Evolution of the fingering pattern of an impacting drop. Phys. Fluids 10 (6), 1359-1374.

VAeZI, M., SeITZ, H. \& YANG, S. 2013 A review on 3D micro-additive manufacturing technologies. Intl J. Adv. Manuf. Technol. 67, 1721-1754.

VillermauX, E. \& Bossa, B. 2011 Drop fragmentation on impact. J. Fluid Mech. 668, 412-435.

Visser, C. W., Pohl, R., Sun, C., Römer, G.-W., Huis in 'T Veld, B. \& Lohse, D. 2015 Toward 3D printing of pure metals by laser-induced forward transfer. Adv. Mater. 27 (27), 4087-4092.

WANG, C.-H., TsaI, H.-L., WU, Y.-C. \& HWAnG, W.-S. 2016 Investigation of molten metal droplet deposition and solidification for 3D printing techniques. J. Micromech. Microengng 26, 095012.

XU, L. 2007 Liquid drop splashing on smooth, rough, and textured surfaces. Phys. Rev. E 75, 056316.

Xu, L., Zhang, W. W. \& Nagel, S. R. 2005 Drop splashing on a dry smooth surface. Phys. Rev. Lett. 94, 184505.

YARIN, A. L. 2006 Dop impact dynamics: splashing, spreading, receding, bouncing ... . Annu. Rev. Fluid Mech. 38 (1), 159-192.

Zhong, S.-Y., QI, L.-H., Luo, J., Zuo, H.-S., Hou, X.-H. \& LI, H.-J. 2014 Effect of process parameters on copper droplet ejecting by pneumatic drop-on-demand technology. J. Mater. Process. Technol. 214 (12), 3089-3097. 